\title{
METHOD OF SMOOTHING PHOTOLUMINESCENCE SPECTRA
}

\author{
O.V. Kovalenko, S.M. Vovk, Ye.G. Plakhtii \\ Oles Honchar Dnipro National University, Dnipro, Ukraine \\ *e-mail: kovalenko.dnu@gmail.com
}

The smoothing method for experimental photoluminescence spectra, which uses Tikhonov regularization method, is proposed. Smoothing problem statement is performed as a statement of the minimization problem based on the least squares criterion. The functional to be minimized is a compound of two terms. The first one is the basic term, which determines the quality of data approximation provided by smooth function. The second one is the regularization term, which sets a constraint on the energy by smooth function derivative of a given order. A solution of the smoothing problem is obtained in the closed form analytically. The solution can be implemented by a simple array of matrix operations; one of those is an operation of matrix inversion. The necessity of matrix inversion imposes constraints on the order of derivative used in the proposed method and on noise level. It is related to the increase of either the order of derivative at the given noise level in data or the noise level at the given order of derivative, that deteriorates the conditionality of a matrix to be inverted and limits the scope of the proposed method, accordingly. Efficiency comparison of the proposed smoothing method, the Savitzky-Golay method, and the moving average method was performed by the numerical simulation of two models of photoluminescence spectra. Their advantages, disadvantages, and also the scopes of applicability are noted. The best values of operating parameters of these methods for the different noise levels in data were obtained. The conditions, under which the potential capabilities of the proposed smoothing method excel the potential possibilities of Savitzky-Golay method, were indicated. The results of smoothing the experimental photoluminescence spectra obtained for $\mathrm{ZnO}$ :Mn nanocrystals and $\mathrm{ZnS}$ :Mn crystals are provided.

Keywords: smoothing of photoluminescence spectra, Savitzky-Golay method, Tikhonov regularization method, photoluminescence spectra, ZnO:Mn nanocrystals, ZnS:Mn crystals.

Received 13.11.2018; Received in revised form 14.12.2018; Accepted 21.12.2018

\section{Introduction}

Photoluminescence (PL) spectroscopy is one of the basic experimental methods of studying the optical properties of dielectric and semiconductor crystals, micro- and nanostructures of materials. The analysis of PL spectra allows to get information about the energy level structure of optically active centers in the band-gap, their activation energy, lifetime of charge carriers in excited state etc. Such information can be obtained on the basis of determining the individual constituent parameters of an experimental PL spectrum $[1,2]$. In practice, the analysis of PL spectra is obstructed by photodetector noise which consists of two basic components: noise depending on the size of the registered signal and thermal noise. Contributions of these noises depend on the photodetector type [3]. Hardware and software methods are usually applied for decreasing the measurement noise level of PL spectra. Hardware methods are based on using different ways of cooling the photoelectronic multiplier and also on application of analog low-frequency filters which perform smoothing the values of electric signal [3]. Software methods are based on smoothing the obtained experimental dependence of PL spectra by the digital filtering methods, where the SavitzkyGolay filtering method occupies the main place traditionally [4]. Undoubtedly, software methods of smoothing are more flexible in application than analog ones. Therefore they are more perspective for the practical usage while they allow to improve and develop smoothing methods.A smoothing method for the registered data of PL is almost always individual for every experimental facility and requires tuning the corresponding operating parameters. It is clear that smoothing of noise at the improper values of operating parameters may lead to the distortion of experimental information and to large errors during its use. Unfortunately, in the discussion of the PL spectrum analysis results it is not often specified how experimental data were smoothed. However, quite often it is possible to see a term "smoothed PL spectrum" in scientific literature [5]. In order to eliminate such disadvantages, we point out the necessity of 
search for the best values of operating parameters of smoothing method. Further we compare the performance of Savitzky-Golay smoothing method and the method based on Tikhonov regularization for two models of PL spectra. The aim of this work is to analyze the efficiency of the mentioned smoothing methods applied to PL spectra as well as to elaborate recommendations for their use.

\section{Problem statement}

Smoothing of any experimental data dependence is the problem of its approximation by a smooth function. The concept of smooth function means that it has a continuous derivative in all the range of its definition. If there are continuous derivatives of a given function up to the $r$-th order inclusive, then we are talking about a smooth function of $r$-th order. In the simplest case, the smoothing problem statement for a known dependence $g(x)$ by a smooth function $f(x)$ can be performed on the basis of the least squares criterion as

$$
\min _{f(x)} \int_{X}|g(x)-f(x)|^{2} d x
$$

where $X$ designates the smoothing interval. The problem statement (1) leads to an obvious solution: $f(x)=g(x) ; x \in X$. Hence, it makes sense only when $g(x)$ is already a smooth function. Unless it is so (for example, the values of function $g(x)$ are distorted by noise), then we need to modify (1) by imposition of limits on the function $f(x)$ to be sought.

The further proposed statement of smoothing problem on the basis of Tikhonov regularization method is obtained from (1) by introducing energy limits on the derivatives of the set orders from the sought function $f(x)$. If the first derivative of the sought function is exposed to such limitation, then the problem (1) is transformed into a problem

$$
\min _{f(x)} \int_{X}|g(x)-f(x)|^{2} d x+\alpha \int_{X}\left|D_{1}[f(x)]\right|^{2} d x
$$

where $D_{1}[f(x)]=\frac{d f(x)}{d x}$ is an operator of differentiation and $\alpha$ is a regularization parameter which sets a compromise between the square error of smoothing and Euclidean norm square of the first derivative of the sought smooth function. If $r$-th derivative of the sought function is exposed to such limitation, then a problem (1) is transformed into a problem:

$$
\min _{f(x)} \int_{X}|g(x)-f(x)|^{2} d x+\alpha \int_{X}\left|D_{r}[f(x)]\right|^{2} d x
$$

where $D_{r}[f(x)]=\frac{d^{r} f(x)}{d x^{r}}$ is an operator of differentiation of $r$-th order. It should be noted that from (3) at $\alpha=0$ we get a trivial solution $f(x)=g(x) ; x \in X$ (when smoothing is not performed) and at $\alpha \rightarrow \infty$ we get a zero solution because a functional (3) does not depend on $g(x)$ at all (if integration coefficients equal zero). Consequently, in this case a useful value $\alpha$ lies in an open interval $(0, \infty)$.

Further, as a measure of efficiency of smoothing method, we use the mean square deviation of the smoothed curve from a true curve which is a mean square error of solution. Thus, the less this error value, the more effective the method is. 


\section{Literature review}

Nowadays, insufficient attention is devoted to the question of smoothing of PL spectra. The smoothed PL spectra are adduced in works [6-8], however information about the way of this smoothing is absent. The works [9-10] perform the decomposition of PL spectra on elementary bands by means of mathematical packages, but smoothing is not shown in details and even the used way of it is not mentioned. There is an opinion in the book [11], which is considered as one of basic sources on the problem of smoothing, that luminescent spectra must be smoothed by Savitzky-Golay method, using the window width that equals 11 or 25 points, and polynomials from quadratic to quintic inclusive. There are smoothed PL spectra in the paper [12], however it is indicated that smoothing was performed by the mathematical packages of qdot.com. The paper [13] shows the smoothed spectra of absorption; besides, it is mentioned, that the data were interpolated by means of splines, but there is no indication of which ones were used. The work [14] adduces the PL spectra smoothed by Savitzky-Golay method with the use of window width of 5 points without mentioning the degree of the smoothed polynomial. The moving average method is applied in paper [15] for smoothing PL spectra without specifying the window width. The work [16] performs smoothing by the moving average method with a window width of 3 points, which is actually a particular case of Savitzky-Golay method. Thus, the analysis of literature review shows that the smoothing methods of PL spectra require the further elaboration and analysis.

\section{Materials and methods}

Savitzky-Golay method is implemented by means of approximation by the sliding polynomial of the given degree. In a discrete case, it can be written as

$$
f_{i}=\frac{\sum_{m=-M}^{M} \omega_{m} g_{i+m}}{\sum_{m=-M}^{M} \omega_{m}}
$$

where coefficients $\omega_{m}$ are determined according to corresponding tables for the given degree of polynomial and window width $M$ [17].

A moving average method is a particular case of (4) and is realized as

$$
f_{i}=\frac{1}{M} \sum_{m=-M}^{M} g_{i+m}
$$

Savitzky-Golay method is characterized by the following complications:

- the greatest degree of polynomial is not always the best; it is regarded that quadratic or cubic polynomials introduce less noise, than polynomials of higher degrees;

- there are some difficulties at the calculation of coefficients on the borders of capture range; it is necessary to calculate its own separate coefficient for every point which cannot become central in a window; accordingly, the more the window, the more difficult calculations must be produced on the borders of measuring.

If it is necessary to obtain smooth derivatives it is recommended to smooth the data before the differentiation procedure. Besides, it is better to apply differentiation four times with less degree of smoothing polynomial, than to get differentiation of fourth order using the polynomial of higher degree of smoothing.

It is possible to write the smoothing method on the basis of Tikhonov regularization (3) for a discrete case as

$$
\min _{f}\left(\|g-f\|^{2}+\alpha\left\|\mathbf{D}_{r} f\right\|^{2}\right)
$$


where vectors $g$ and $f$ describe given and sought data and matrix $\mathbf{D}_{r}$ responds to operator $D_{r}$. The problem (6) has an obvious solution

$$
f=\left(\mathbf{I}+\alpha \mathbf{D}_{r}^{T} \mathbf{D}_{r}\right)^{-1} g
$$

where $\mathbf{I}$ is an identical matrix and $\mathbf{D}_{r}^{T}$ is a transposed matrix $\mathbf{D}_{r}$.

The operator of calculating the zeroth order derivative $r=0$ is described by the diagonal unit matrix $\mathbf{D}_{0}=I$ with dimension $N \times N$. It is easy to obtain derivative matrix definition for any $r$ order. However, it should be mentioned that the matrices $D_{r}^{T} D_{r}$ and $A^{T} A$ have the $N \times N$ size.

Thus, the offered smoothing method consists of the implementation of simple matrix operations corresponding to (7).

\section{Experiments and results}

For the demonstration of possibilities of offered smoothing methods, procedures of two noisy synthesized PL spectra were carried out. Each of them consisted of five elementary Gaussian bands. One of them had essential overlapping of elementary bands and the other had inessential one; as a result, maxima of separate elementary bands were shown in it. A modeling problem was to imitate noise, which is characteristic of spectrum measurements, in the synthesized PL spectra and to find the optimal smoothing methods. Thus, parameter $r=5$ was fixed for Tikhonov method and parameter $\alpha$ was the only subject to adjustment. All calculations were performed in the shell of Matlab for 100 realizations of noise.

The PL spectrum, where elementary bands were shown visually, was modeled in the range of wavelengths of $400-650 \mathrm{~nm}$ with $\Delta \lambda=0.5 \mathrm{~nm}$ step which responded to $N=501$ discrete counting. A spectrum model was intended for the imitation of real PL spectrum of $\mathrm{ZnO}: \mathrm{Mn}$ nanocrystals and was set by the sum of five Gaussian functions with parameters: $A_{1}=1000, \quad m_{1}=80 \Delta \lambda, \quad \sigma_{1}=20 \Delta \lambda ; \quad A_{2}=900, \quad m_{2}=30 \Delta \lambda, \quad \sigma_{2}=20 \Delta \lambda$; $A_{3}=700, m_{3}=140 \Delta \lambda, \sigma_{3}=30 \Delta \lambda ; A_{4}=200, m_{4}=200 \Delta \lambda, \sigma_{4}=30 \Delta \lambda ; A_{5}=100$, $m_{5}=250 \Delta \lambda, \sigma_{5}=30 \Delta \lambda$ where $A_{i}, m_{i}$, and $\sigma_{i}$ are an amplitude in relative units, position of maximum, and standard deviation of $i$-th Gaussian function in the discrete counting. The constant background of amplitude $A_{0}=50$ and noise imitating the noise of photoelectronic multiplier were added as a sum of noise, which depends on a signal, and thermal noise to the sum of Gaussian functions. This noise was modeled by the correlation $\xi_{i}=\left|g\left(x_{i}\right)\right|^{1 / 2} \eta_{i}+15 \varsigma_{i}$ where $\eta_{i}$ and $\varsigma_{i}$ are the values of Gaussian random quantities with zero mathematical expectation and unit variance. The same model was used in the work [18].

The PL spectrum, where maxima of elementary bands are not shown because of strong overlapping of elementary bands, was modeled in the energy range of 1.722-2.582 eV with step $\Delta \lambda=1.265 \cdot 10^{-3} \mathrm{eV}$, which responded to $N=681$ discrete counting. A spectrum model was intended for the imitation of the real PL spectrum of ZnS:Mn crystals and was set by the sum of five Gaussian functions with parameters: $A_{1}=1000, m_{1}=240 \Delta \lambda, \sigma_{1}=40 \Delta \lambda$; $A_{2}=450, m_{2}=297 \Delta \lambda, \sigma_{2}=40 \Delta \lambda ; A_{3}=350, m_{3}=216 \Delta \lambda, \sigma_{3}=40 \Delta \lambda ; A_{4}=250$, $m_{4}=180 \Delta \lambda, \sigma_{4}=40 \Delta \lambda ; A_{5}=150, m_{5}=335 \Delta \lambda, \sigma_{5}=40 \Delta \lambda$ where $A_{i}, m_{i}$, and $\sigma_{i}$ are an amplitude, position, and standard deviation of the $i$-th Gaussian function. The constant background of amplitude $A_{0}=50$ and noise were added to the sum of Gaussian functions. 
The noise was described by the correlation $\xi_{i}=\left|g\left(x_{i}\right)\right|^{1 / 2} \eta_{i}+15 \varsigma_{i}$ where $\eta_{i}$ and $\varsigma_{i}$ are the values of Gaussian random quantities with zero mathematical expectation and unit variance.

The results of smoothing of synthesized PL spectra are shown in Fig. 1.
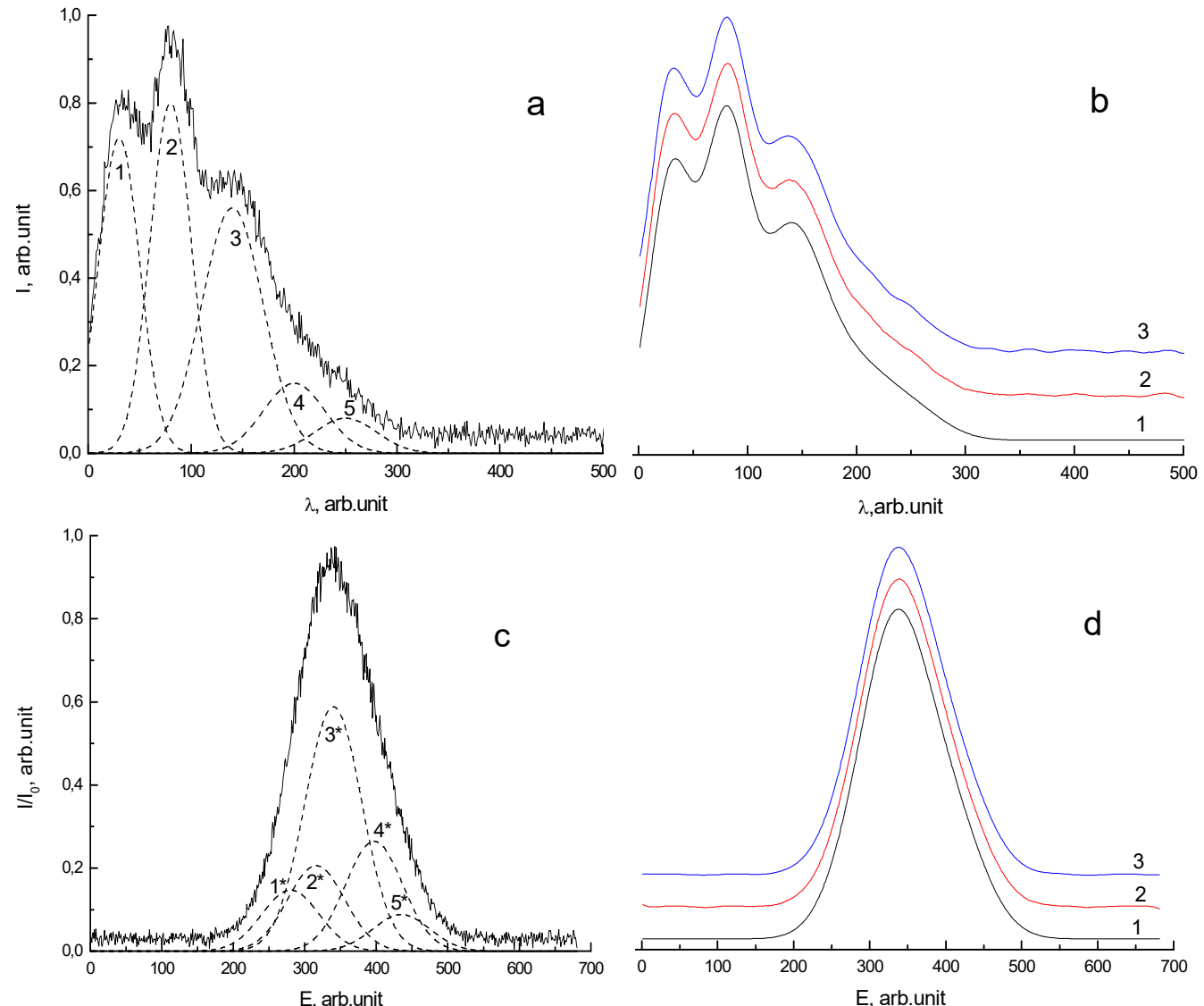

Fig. 1. The results of modeling: a) the sum of five Gaussian curves (1-5) with pedestal and adding of thermal noise and noise depending on a signal; b) the initial integral curve (1), smoothed by Savitzky-Golay method with the least total error (2), smoothed by Tikhonov method with the least total error (3); c) the sum of five Gaussian curves $\left(1^{*}-5^{*}\right)$ with permanent background and adding of thermal noise and noise depending on a signal; d) the initial integral curve (1), smoothed by Savitzky-Golay method with the least total error (2), smoothed by Tikhonov method with the least total error (3).

The curves 1, 2, 3 are shifted along the vertical axes for visualization in the Fig. 1b and Fig. 1d.

The initial PL spectrum, which consists of a sum of five Gaussian curves with a permanent background and noise including the sum of thermal one and noise depending on a signal, is shown in Fig. 1a. The degree of overlapping of elementary bands is small in this PL spectrum. For this case $\Delta m \geq 2.2 \sigma$ for elementary bands with approximately identical amplitudes [19] where $\Delta m$ is a distance between maxima of individual bands, which are side by side, $\sigma$ is a standard deviation of the widest individual Gaussian band. In Fig. 1b there are an initial integral curve (1); the one smoothed by Savitzky-Golay method (2) with the use of polynomial of seventh degree with an optimal window of 81 discrete counting length and minimum total error that equals 16491 standard units; the curve smoothed by Tikhonov method (3) with a minimum total error that equals 18745 standard units with the regularization coefficient $\alpha=2.5 \cdot 10^{7}$. At smoothing by moving average method and 
optimal window that equals 13 discrete counting, a minimum total error equals 2805 standard units.

The initial PL spectrum, which consists of a sum of five Gaussian curves with a permanent background and noise including the sum of thermal one and noise depending on a signal, is shown in Fig. 1c. The degree of overlapping of elementary bands was large in this PL spectrum. For this case the difference in position of maxima of individual bands, which are side by side, has a size $\Delta m<2.2 \sigma$ for $A_{i} \approx A_{i+1}$ [19]. In Fig. $1 \mathrm{~d}$ there are an initial integral Gaussian curve with a permanent background (1); the one smoothed by SavitzkyGolay method (2) with the use of polynomial of seventh degree with an optimal window 191 discrete counting length and minimum total error that equals 11013 standard units; the curve smoothed by Tikhonov method (3) with a minimum total error that equals 9624 standard units at the parameter of regularization $\alpha=5.6 \cdot 10^{8}$. At smoothing by moving average method and optimal window that equals 20 discrete counting, a minimum total error equals 24558 standard units.

The performed modeling showed that for both modeled spectra and correlation signal/noise that equals 10 the maximally attainable order of polynomial in Savitzky-Golay method was equal to seven, and the maximally attainable order of derivative in the offered method on the basis of Tikhonov regularization was equal to five. For a correlation signal/noise that equals 100 , the maximally attainable order of polynomial in Savitzky-Golay method was equal to seven and the order of derivative in an offered method was equal to six. For a correlation signal/noise that equals 1000 , the maximally attainable order of polynomial in Savitzky-Golay method was also equal to seven and the order of derivative in the offered method was equal to six.

The performed modeling shows that at the strong overlapping of elementary bands the Tikhonov method provides the best results of smoothing, while at the weak overlapping of elementary bands the best results are provided by Savitzky-Golay method.

As it was said above, the synthesized PL spectra model the PL spectrum of ZnO:Mn nanocrystals shown in the paper [20] and also the PL spectrum of ZnS:Mn crystals, which elementary bands were analyzed in the paper [21] - Fig. 2.
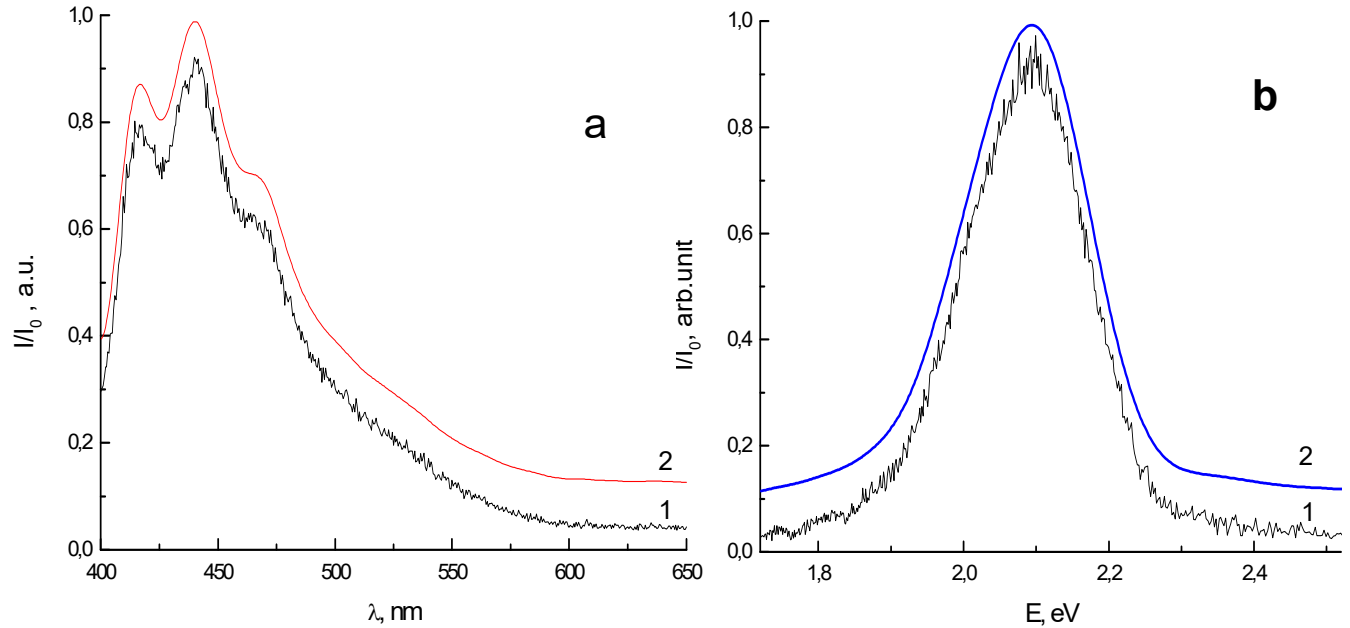

Fig. 2. Results of the experiments with smoothing of PL spectra:

a) experimental PL spectrum of $\mathrm{ZnO}$ :Mn crystals from [20] (curve 1) and the result of its smoothing by Savitzky-Golay method (curve 2); b) experimental PL spectrum of ZnS:Mn crystals (curve 1) and the result of its smoothing by Tikhonov method for $r=5$ (curve 2). Curves 2 are shifted relative to curves 1 along the vertical axes for visualization. 
There are five elementary Gaussian bands in these PL spectra, which are adduced according to data of works [19, 21]. In Fig. 2a, there are an experimental PL spectrum and one smoothed by Savitzky-Golay method on the basis of polynomial of seventh degree and window-length of 81 point. There are an experimental PL spectrum and one smoothed by Tikhonov method with the regularization coefficient $\alpha=5.6 \cdot 10^{8}$ in Fig. $2 \mathrm{~b}$. The data in Fig. 2 show the efficiency of the used smoothing methods and chosen values of their parameters.

\section{Conclusions}

A numerical modeling shows the smoothing efficiency of PL spectra both by means of the offered method based on Tikhonov regularization and by means of Savitzky-Golay method. Application of moving average method did not provide smoothing of spectra with adequate accuracy. The performed modeling shows that at the strong overlapping of elementary bands of emission ( $\Delta m<2.2 \delta$ for $A_{i} \approx A_{i+1}$ ) it is recommended to use Tikhonov method for PL spectrum smoothing. At the weak overlapping of elementary bands of emission ( $\Delta m \geq 2.2 \delta$ for $A_{i} \approx A_{i+1}$ ) it is recommended to use Savitzky-Golay method for PL spectrum smoothing.

At application of Savitzky-Golay method for smoothing the PL spectra obtained by means of traditional photoelectronic multipliers, it is expedient to use a polynomial not higher than seventh degree with the window width, which is no more than one third part of the measurement range. At application of the offered method based on Tikhonov regularization, it is expedient to use a derivative which order is not higher than fifth or sixth. In both cases it is recommended to perform adjustment of operating parameters of these methods both for a current noise environment, which is conditioned by noise of used photoelectronic multipliers, and for the maximal value of useful spectrum amplitudes. The smoothing of experimental PL spectrum by the offered method and Savitzky-Golay method are identically successful in coordinates $I(\lambda)$ and $I(E)$

\section{References}

1. Yang, H. Obtaining information about protein secondary structures in aqueous solution using Fourier transform IR spectroscopy / H. Yang, S. Yang, J. Kong, A. Dong \& S. Yu// Nature protocols.- 2015. - Vol. 10, No. 3. - P. 382 - 396.

2. Bacherikov, Y.Y. Competition of the self-activated and Mn-related luminescence in ZnS single crystals / Y.Y. Bacherikov, I.P. Vorona, I.V. Markevich, N.O. Korsunska, R.V. Kurichka // Solid State Communications. - 2018. - Vol. 274. - P. 31 - 35.

3. Ren, M.Y. Design of Pre-Amplifiers for Photoelectric Detector / M.Y. Ren, L. Tian, W. Wang, X. W. Liu, Z.G. Mao// In Applied Mechanics and Materials, Trans Tech Publications. - 2013. - Vol. 380. - P. 3308 - 3311.

4. Schafer, R.W. What is a Savitzky-Golay filter?[lecture notes] / R.W. Schafer // IEEE Signal processing magazine. -2011. - Vol. 28, No 4. - P. $111-117$.

5. Wang, M. Photoluminescence of Si-rich silicon nitride: Defect-related states and silicon nanoclusters / M. Wang, D. Li, Z. Yuan, D. Yang, D. Que // Applied physics letters. 2007.- Vol. 90, No. 13. - P. 131903.

6. Srivastava, A. Valley Zeeman effect in elementary optical excitations of monolayer $\mathrm{WSe}_{2} /$ A. Srivastava, M. Sidler, A.V. Allain, D.S. Lembke, A. Kis, A. Imamoğlu //Nature Physics. - 2015. - Vol. 11, No. 2.- P. 141.

7. Collins, S. Radiative recombination mechanisms in CdTe thin films deposited by elemental vapor transport / S. Collins, S. Vatavu, V. Evani, M. Khan, S. Bakhshi, V. Palekis, C. Rotaru, C. Ferekides //Thin Solid Films. -2015. - Vol. 582.- P. 139 - 45. 
8. Thandavan, T.M. Photoluminescence properties of un-doped and Mn-doped $\mathrm{ZnO}$ nanostructures / T.M. Thandavan, C.S. Wong, S.M. Gani, R.M. Nor //Materials Express. -2014. - Vol. 4, No. 6. - P. 475 - 82.

9. Untila, D. Photoluminescence properties of lamellar nano-composites obtained by $\mathrm{Cd}$ intercalation of GaSe and GaSe: Eu single crystals / D. Untila, V. Cantser, M. Caraman, I. Evtodiev, L. Leontie, L. Dmitroglo // Physica status solidi (c) . -2015. - Vol. 12, No. 1-2. P. $65-69$.

10. Reddy, P.R. Morphological analysis and photoluminescence properties of hydrophilic porous anodic alumina formed in oxalic acid [Text] / P.R. Reddy, K.M. Ajith, N.K. Udayashankar // Journal of Materials Science: Materials in Electronics. -2016 . - Vol. 27, No. 5.- P. 5331-44.

11. G. Talsky, Derivative spectroscopy: low and higher order, 1994. VCH Publishers: 1-228.

12. Bouzidi, M. Time-resolved photoluminescence and photoreflectance spectroscopy of GaN layers grown on SiN-treated sapphire substrate: Optical properties evolution at different growth stages/ M. Bouzidi, S. Soltani, Z. Chine, A. Rebey, M.K. Shakfa //Optical Materials. -2017. - Vol. 73.- P. 252-9.

13. Yuan, H. Gold nanostars: surfactant-free synthesis, 3D modelling, and two-photon photoluminescence imaging/ H. Yuan, C.G. Khoury, H. Hwang, C.M. Wilson, G.A. Grant, T. Vo-Dinh //Nanotechnology. -2012.- Vol. 23, No. 7.- P. 075102.

14. Praveena, R. Surface plasmon resonance, photoluminescence and surface enhanced Raman scattering behaviour of $\mathrm{Ag} / \mathrm{ZnO}, \mathrm{ZnO} / \mathrm{Ag}$ and $\mathrm{ZnO} / \mathrm{Ag} / \mathrm{ZnO}$ thin films/ R. Praveena, V.S. Sameera, M.A. Mohiddon, M.G. Krishna // Physica B: Condensed Matter.- Vol. 555

15. Zuo, D. Diffusion characterization using electron beam induced current and timeresolved photoluminescence of InAs/InAsSb type-II superlattices. InCLEO/ D. Zuo, R. Liu, D. Wasserman, J. Mabon, Z. He, S. Liu, Y.H. Zhang, E.A. Kadlec, B.V. Olson, E.A. Shaner // Science and Innovations. - 2015.- P. SM2G-4

16. Tarelkin, S.A. Evidence of linear Zeeman effect for infrared intracenter transitions in boron doped diamond in high magnetic fields/ S.A. Tarelkin, V.S. Bormashov, S.G. Pavlov, D.L. Kamenskyi, M.S. Kuznetsov, S.A. Terentiev, D.D. Prikhodko, A.S. Galkin, H.W. Hübers, V.D. Blank // Diamond and Related Materials. - 2017. - Vol. 75. - P. 52-57.

17. Madden, H.H. Comments on the Savitzky-Golay convolution method for leastsquares-fit smoothing and differentiation of digital data/ H.H. Madden // Analytical chemistry.-1978.- Vol. 50, No. 9.- P.1383-6.

18. Vovk, S.M. Method for numerical differentiation of noisy data with outliers/ S.M. Vovk // Radio Electronics, Computer Science, Control. - 2017. - No. 3. - P.44 - 52.

19. Kovalenko, A.V. Application of derivative spectroscopy method to photoluminescence in ZnS: Mn nanocrystals/ A.V. Kovalenko, Ye.G. Plakhtii, S.M. Vovk// Ukrainian Journal of Physical Optics. - 2018.- Vol. 19, No. 3. .- P.133-140.

20. Bulaniy, M.F. Synthesis of $\mathrm{ZnO}$ and $\mathrm{ZnO}$ : Mn Nanopowders by Ultrasonic Spray Pyrolysis/ M.F. Bulaniy, V.Y. Vorovsky, A.V. Kovalenko, O.V. Khmelenko// Journal of Nano- and Electronic Physics. - 2016. - Vol. 8, No. 2.- P. 2043.

21. Bulaniy, M.F. Obtaining of nanocrystals $\mathrm{ZnS}$ : Mn by means of self-propagating high-temperature synthesis/ M.F. Bulaniy, A.V. Kovalenko, A.S. Morozov, O.V. Khmelenko // Journal of Nano-and Electronic Physics. - 2017 . - Vol. 9, No. 2.- P. 2007 - 1 - 4. 